\title{
Online Free Walking Trajectory Generation for Biped Humanoid Robot KHR-3(HUBO)
}

\author{
Ill-Woo Park, Jung-Yup Kim, Jungho Lee and Jun-Ho Oh \\ Humanoid Robot Research Center (N9), Department of Mechanical Engineering \\ Korea Institute of Science and Technology (KAIST) \\ 373-1 Guseong-dong, Yuseong-gu, Daejeon 305-701, Republic of Korea \\ Email: \{mrquick|kirk1|jungho77\}@hubolab.kaist.ac.kr,jhoh@kaist.ac.kr
}

\begin{abstract}
This paper describes an algorithm about online gait trajectory generation method, controller for walking, brief introduction of humanoid robot platform KHR-3 (KAIST Humanoid Robot - 3: HUBO) and experimental result. The gait trajectory has continuity, smoothness in varying walking period and stride, and it has simple mathematical form which can be implemented easily. It is tested on the robot with some control algorithms.

The gait trajectory algorithm is composed of two kinds of function trajectory. The first one is cycloid function, which is used for ankle position in Cartesian coordinate space. Because this profile is made by superposition of linear and sinusoidal function, it has a property of slow start, fast moving, and slow stop. This characteristics can reduce the over burden at instantaneous high speed motion of the actuator. The second one is $3^{\text {rd }}$ order polynomial function. It is continuous in the defined time interval, easy to use when the boundary condition is well defined, and has standard values of coefficients when the time scale is normalized. Position and velocity values are used for its boundary condition. Controllers mainly use $F / T$ (Force/Torque) sensor at the ankle of the robot as a sensor data, and modify the input position profiles (in joint angle space and Cartesian coordinate space). They are to reduce unexpected external forces such as landing shock, and vibration induced by compliances of the sensors and reduction gears, because they can affect seriously on the walking stability.

This trajectory and control algorithm is now on the implementing stage for the free-walking realization of KHR-3. As a first stage of realization, we realized the marking time and forward walking algorithm with variable frequency and stride.
\end{abstract}

Index Terms - HUBO, Humanoid, Biped walking, Trajectory planning

\section{INTRODUCTION}

The research in humanoid robot is now on its way of diverging into various categories. The research on such areas as artificial intelligence, hardware development, realization of biped locomotion, and interaction with the environment are gaining a rapid phase of development with the help of the rapid growth of technology. The research on humanoid robots has gained a particular interest in this new phase as humanoids tend to change the concept of the robot. In the past, robots were confined to the industry carrying out such jobs as welding, and parts-assembly (automobile and electronic devices) in that the objectives, specification and optimal design parameters were clearly defined with concern to the economic aspects, productivity and efficiency. As the economical paradigm is changing from mass production to small quantity batch production, people's concept of the robot has been gradually diverging. By today, it has come to a situation, where the robot should be able to perform a wide variety of functions that helps people in their daily life.

The important features of biped humanoid robots are the human-like shape and movements. The robot always gets the expectation of people that this type of robot can walk and move like a human. It is also expected that it can climb up stairs, walk on uneven terrain, run, and dance. Many researches now show the realization of them.

Honda humanoid [1] [3], WABIAN series of Waseda University [4],[5], H6,7 of Tokyo University [6],[7], HRP of AIST [8],[9] and JOHNNIE [9] are the well known human scale biped humanoid robots. Mostly, the dynamic walking control strategy of biped robots is based on the walking pattern generation and modification, which considers the stable ZMP (Zero Moment Point) trajectory and online balance control. We made walking trajectory first, and its validity is proved by experiment with the real platform. This research process has been done in all KHR [10] [12] series.

Several online controllers based on the sensory feedback are required because the actual ZMP trajectory is different from the desired ZMP trajectory due to reasons such as the unevenness of the surface, sensing errors, and imperfect dynamic model of the robot. Takanishi et al. have studied about online walking pattern generation and walking stabilized control by using upper body motion based on the ZMP information [4],[5]. Kajita et al. introduced a method of the biped walking pattern generation by using a preview control of ZMP that uses future reference [8],[9]. Kagami et al. used a torso position compliance control method to track a given ZMP trajectory [6],[7].

We describe a novel gait trajectory method for online free walking pattern generation method, controllers for stabilization using F/T sensor, a brief introduction of the robot platform KHR-3 (HUBO), and its simulation and experimental result (marking time mode with variable walking period) are presented in this paper. The gait trajectory algorithm uses simple and continuous functions which can be easily implemented on the real robot. We can measure the ground reaction force, moment, and ZMP directly. The controller algorithm which modifies the trajectory stabilizes the robot by utilizing the data. 


\section{ONLINE GAIT GENERATION \\ A. Coordinate frame for gait trajectory}

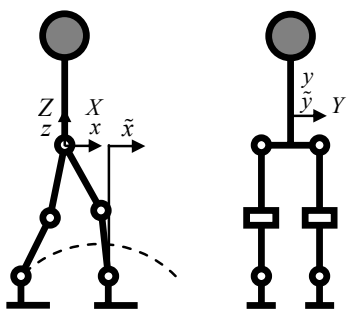

Fig. 1 Coordinate frame for gait trajectory

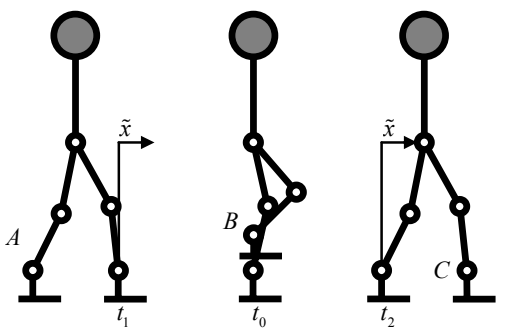

Fig. 2 Sagittal plane view of coordinate frame
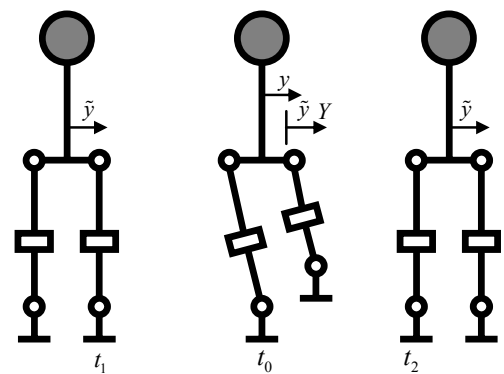

Fig. 3 Coronal plane view of coordinate frame

Fig. 1 shows the coordinate system. $x-y-z$ is local coordinate frame which is located on the pelvis center and $\mathrm{X}$ $\mathrm{Y}-\mathrm{Z}$ is global coordinate frame, which has same origin initially. Walking pattern for lower body is generated on the basis of these coordinate frames. We made three position parameters, left/right ankle position and pelvis center position. Those position parameters are implemented to the robot after coordinate transformation onto $x-y-z$ coordinate frame. Fig. 2 shows coordinate frame for forward walking. $\tilde{x}$ is located on the front supporting foot during the other leg swings in $x$ direction. $t_{1}$ is the start time of swing from DSP (Double Support Phase) and $t_{2}$ is the end time of swing to the other DSP. If $t_{0}$ exists, $t_{0}$ is that the swing ankle and pelvis center coincide with the supporting ankle in $x$ direction. We make the pelvis center $-x$ trajectory to have the position be in the center of two ankles at $t_{1}$ and $t_{2}$. First, we generated the pelvis and the swing leg trajectory in this local coordinate frame - $\tilde{x}$, and implemented to the robot on the basis of $x$ frame by translating the coordinate frame. Fig. 3 shows $y$ and $\tilde{y}$ coordinate frame. $\tilde{y}$ is on the ground and has same direction with $y$. We make pelvis $-y$ trajectory on the $\tilde{y}$ frame and translate it onto $y$ frame.

\section{$B$. Trajectory generation on sagittal plane ( $X$ - direction)}

We use cycloid function for swing leg ankle position, and $3^{\text {rd }}$ order polynomial interpolation for pelvis center in $x$ direction. For simplicity, we describe the trajectories with respect to $\tilde{x}$-coordinate frame. Equation (1) and (2) are the trajectory equations of the swing leg ankle position and the pelvis center position.

$$
\begin{aligned}
& \text { Ank_ } \tilde{x}(t)=(b+f)\left(\frac{t-t_{1}}{t_{2}-t_{1}}-\frac{1}{2 \pi} \sin \left(2 \pi \frac{t-t_{1}}{t_{2}-t_{1}}\right)\right)-b \\
& P_{-} \tilde{x}(t)=a_{3}\left(\frac{t-t_{1}}{t_{2}-t_{1}}\right)^{3}+a_{2}\left(\frac{t-t_{1}}{t_{2}-t_{1}}\right)^{2}+a_{1}\left(\frac{t-t_{1}}{t_{2}-t_{1}}\right)+a_{0}
\end{aligned}
$$

Where, $b$ and $f$ are the swing ankle positions with respect to the coordinate frame $\tilde{x}$ when the time $t$ is $t_{1}$ and $t_{2} . a_{i}(i=0$, $1,2,3)$ are polynomial coefficients which can be found by the boundary conditions as following.

The boundary conditions of $P_{-} \tilde{x}(t)$ are fallowed as, $P_{-} \tilde{x}\left(t_{1}\right)=-b / 2, P_{-} \tilde{x}\left(t_{2}\right)=f / 2, \dot{P}_{-} \tilde{x}\left(t_{1}\right)=\alpha b, \dot{P}_{-} \tilde{x}\left(t_{2}\right)=\alpha f$. If $t_{0}$ exists, boundary conditions and time intervals are slightly modified. The time intervals are divided into $t_{1} \leq t \leq t_{0}$ and $t_{0} \leq t \leq t_{2}$, the trajectory conditions at time $t_{0}$ are $P_{-} \tilde{x}\left(t_{0}\right)=0$ and $\dot{P}_{-} \tilde{x}\left(t_{0}\right)=\beta|f-b|$. Where, $\alpha$ and $\beta$ are constant coefficients. Those conditions mean that the pelvis center position, when $t$ is $t_{1}, t_{2}$ and $t_{0}$, is on the center of two ankles, and their velocities (at $t=t_{1}, t_{2}$, and $t_{0}$ ) are in proportion to the displacement between ankles. This condition preserves the trajectory continuity between the end of current step and the start of next step. We can find out the value of $t_{0}{ }^{*}$ from the condition that two ankles are coincided in $\tilde{x}$-direction at $t_{0}$.

$$
t_{0}=\frac{t_{2}-t_{1}}{2 \pi} \cos ^{-1}\left(1-2 \sqrt{\left(1-\left(\frac{2 b}{b+f}-1\right)^{2}\right)}\right)+t_{1}
$$

The supporting front ankle and swing ankle position profiles can be calculated by simple coordinate translation.

C. Trajectory generation on coronal plane ( $y$-direction)

We use $3^{\text {rd }}$ order polynomial interpolation method as a pelvis center trajectory in $\tilde{y}$ frame.

$$
\begin{gathered}
P_{-} \tilde{y}(t)=\bar{a}_{3}\left(\frac{t-t_{1}}{t_{2}-t_{1}}\right)^{3}+\bar{a}_{2}\left(\frac{t-t_{1}}{t_{2}-t_{1}}\right)^{2}+\bar{a}_{1}\left(\frac{t-t_{1}}{t_{2}-t_{1}}\right)+\bar{a}_{0} \\
\text { Where } \bar{a}_{i}(\mathrm{i}=0,1,2,3) \text { is polynomial coefficient. }
\end{gathered}
$$

\footnotetext{
"Equation (1) is a cycloid function. This type of function is a part of ellipsoidal equation written in parametric form.
} 
The boundary conditions of (4) are similar to (3) case. We made the pelvis sway amplitude to have a peak value at $t=(t 1+t 2) / 2$. Their boundary conditions at $t_{1},(t 1+t 2) / 2$ and $t_{2}$ are $P_{-} \tilde{y}\left(t_{1}\right)=0, P_{-} \tilde{y}\left(\left(t_{2}+t_{1}\right) / 2\right)=-S y, P_{-} \tilde{y}\left(t_{2}\right)=0, \dot{P}_{-} \tilde{y}\left(t_{1}\right)=-\alpha_{1}$, $\dot{P}_{-} \tilde{y}\left(\left(t_{2}+t_{1}\right) / 2\right)=0$, and $\dot{P}_{-} \tilde{y}\left(t_{2}\right)=-\alpha_{1}$. Where, $S y$ and $\alpha_{1}$ are sway amplitude and velocity of pelvis at the time. We assume that the value of $\alpha_{1}$ is constant because of the trajectory continuity. The sign of $S y$ and $\alpha_{1}$ is to be changed by lifting up the other leg.

We use the $1-\cos ()$ velocity profile function as $y$-direction ankle position. This means whatever the body center trajectory to be, we can add it as shown in Fig. 4 and its position trajectory has a shape of cycloid function.

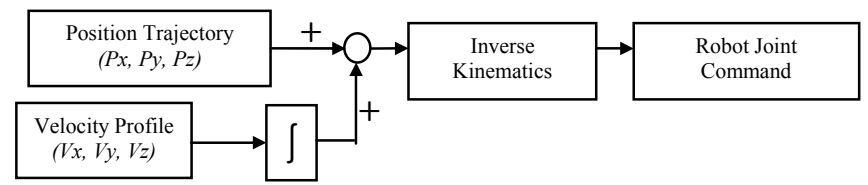

Fig. 4 Trajectory implementation block diagram

This gait generation method preserves its continuity and differentiability with the change of walking cycle and stride. Fig 5 . shows the simulation result. Where, $t_{i}^{j}$ denotes $t_{i}$ time at $j$-th step $^{\dagger}$ and the other values are $b_{1}=100, f_{1}=400, b_{2}=400$, $f_{2}=200$. This figure is described in the global $\mathrm{X}-\mathrm{Y}-\mathrm{Z}$ coordinate frame.

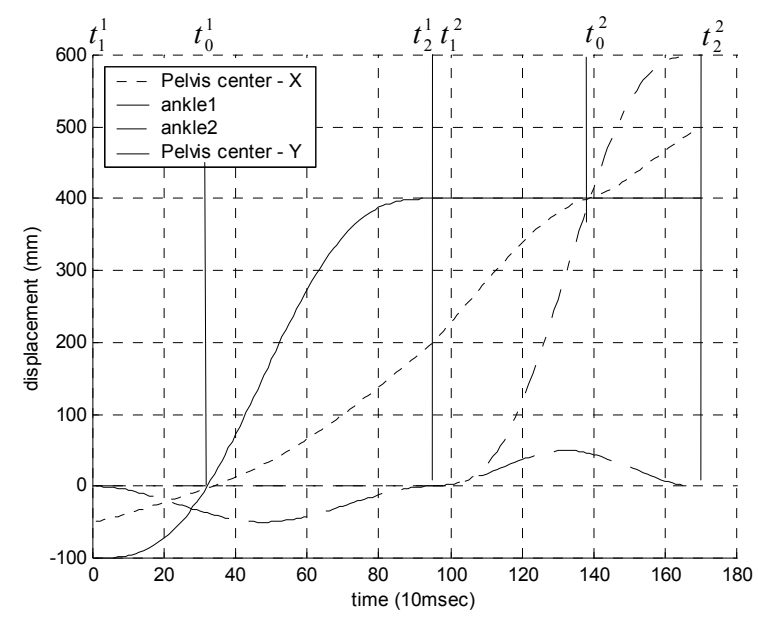

Fig. 5 Profile simulation sample ( $t_{0}$ exists)

When $b \cdot f^{*}$ is negative, $t_{0}$ does not exist. This means the swing foot (shown in Fig. 6: ankle1) does not cross over the supporting foot.

This gait generation method has following merits.

1) Continuity: This gait generation method can make smooth trajectories in any walking period and $\mathrm{X}-\mathrm{Y}$ direction

\footnotetext{
We can adjust the DSP ratio by setting the value $t_{2}^{j}$ and $t_{1}^{j+1}$ differently.

$\$ b$ and $f$ are all positive in forward walk and all negative in backward walk.
}

stride. It is because the gait is generated by combination and superposition of smooth functions with proper conditions. We have the experimental result with variable walking period in marking time mode in section $\mathrm{V}$.

2) Simplicity: It is easy to implement into the biped robot, because we use $3^{\text {rd }}$ order interpolation trajectory and cycloid function and we can simply update the boundary conditions and step period in every step.

3) Decoupled property: The trajectory on each axis X-Y$\mathrm{Z}$ is decoupled from each other.

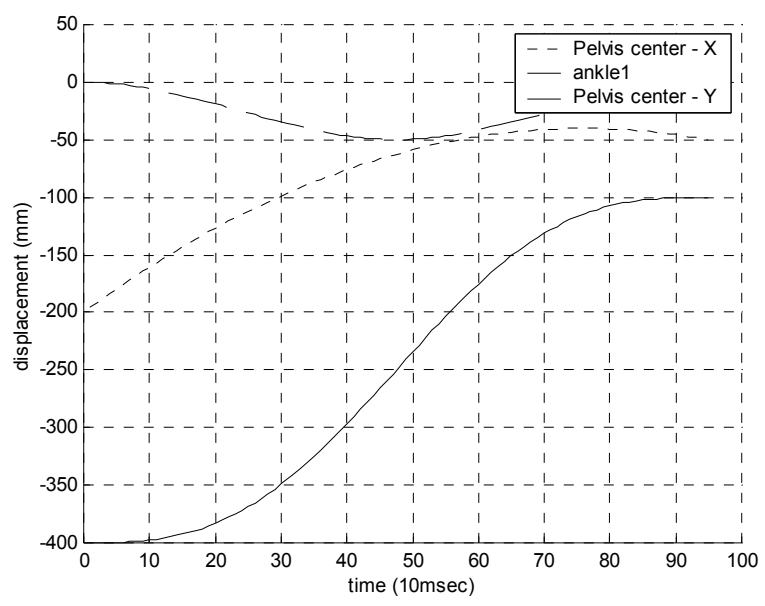

Fig. 6 Profile simulation sample ( $t_{0}$ does not exist)

\section{CONTROLLER}

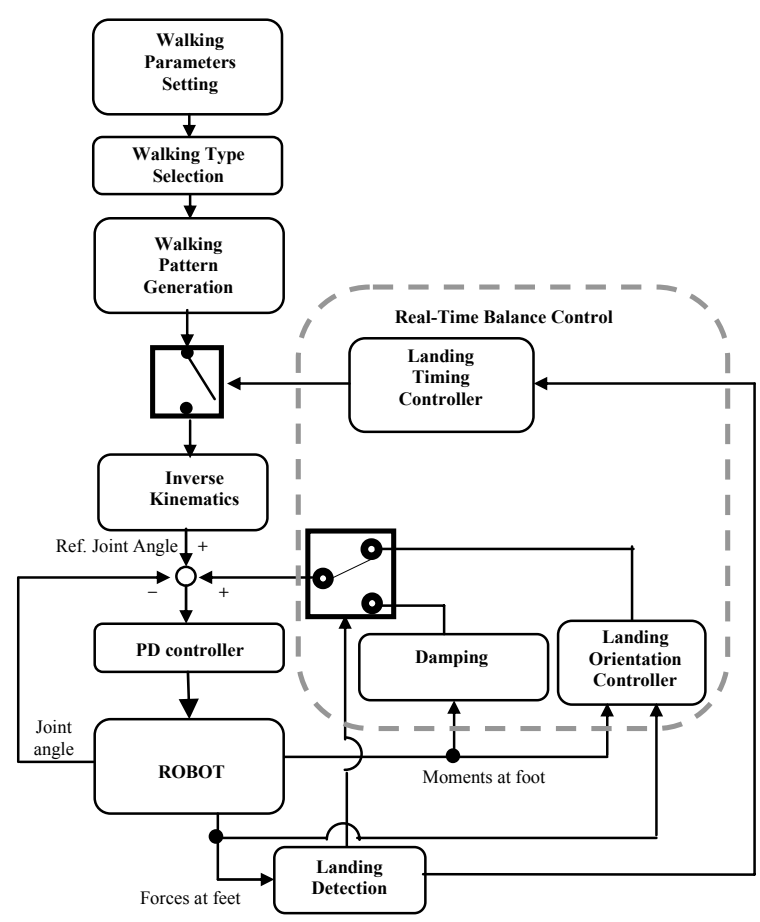

Fig. 7 Controller block diagram for trajectory implementation 
Fig. 7 shows overall control architecture for experiment. All joints of the robot are controlled by PD servo controller. After we generate the walking patterns and joint angles described in previous section, we modify them with controllers by sensory feedback. We control the ankle joint angles by using $\mathrm{F} / \mathrm{T}$ sensors which can detect the ground reaction forces and moments. Those controllers can reduce the oscillations and shocks induced by abnormal contact of the foot by modifying the original angle trajectory. Those are enabled and disabled by switching (as shown in Fig. 7 and 11) on the base of gait pattern and $\mathrm{F} / \mathrm{T}$ sensor data.

\section{A. Controllers for walking}

We use following controllers for walking experiment.

1) Damping: The vibration of whole body in SSP (Single Support Phase) is mainly caused by the compliance of F/T sensor and harmonic drive reduction gear at ankle. We modeled the robot in SSP as the inverted pendulum with compliant joint as shown in Fig. 8.

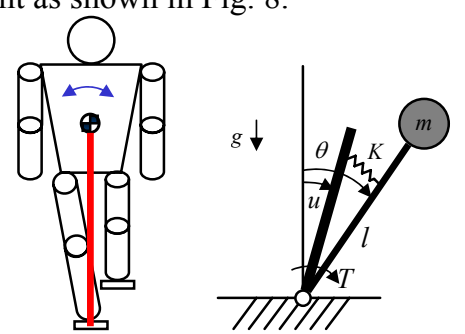

Fig. 8 Inverted pendulum model with compliant joint in SSP

$$
\begin{aligned}
& T= m g l \theta-m l^{2} \ddot{\theta}=K(\theta-u) \\
& u_{m}=u-k_{d} \hat{\dot{\theta}}
\end{aligned}
$$

The equation of motion and the control law are (5) and (6).

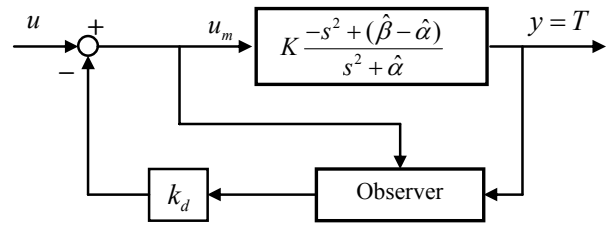

where, $T$ : measured torque, $\hat{\alpha}: K / m l^{2}-g / l, \hat{\beta}: K / m l^{2}, k_{d}$ : damping control gain, and $u_{m}$ : ankle joint angle controller input.

Fig. 9 Damping controller block diagram

2) Landing orientation: This controller integrates the measured torque over time to achieve soft landing and stable contact by adapting the ankle joints to the ground surfaces (Fig. 10) inclination. It makes the ankle joint to be compliant during short period at landing. The control law of the landing orientation control is (7).

$$
u_{m}=u+\frac{T(s)}{C_{L} s+K_{L}}
$$

where, $C_{L}$ : damping coefficient, $K_{L}$ : the stiffness, $u$ : the reference ankle joint angle, and $u_{m}$ : the compensated reference ankle joint angle.

3) Landing timing: The landing timing controller prevents the robot from being unstable during landing by the modification of walking pattern schedule. That is, if the foot does not land on the ground at the end of stretch at the prescribed schedule, the time scheduler of the main computer will halt the motion flow until the foot contacts the ground. Therefore, the real walking motion can follow the prescribed walking pattern despite anomalies emerging during walking.

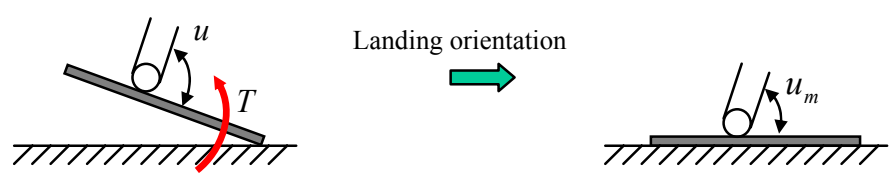

Fig. 10 The landing orientation control

Fig. 12 shows controller activation timing diagram. The damping controller which controls roll and pitch angle of the ankles shown in Fig. 11 by using F/T sensor is activated in supporting phase and recovered in swing phase. Cartesian coordinate controller denotes that the control command acts on the position of ankles (landing timing controller) and pelvis (ZMP) in this paper. ZMP controller is activated in DSP at stop stage.

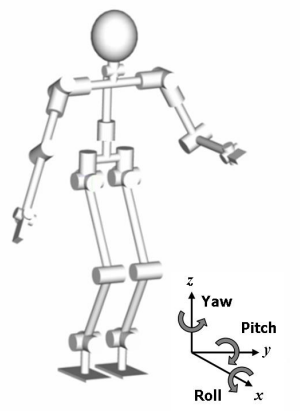

Fig. 11 Schematic of KHR-3

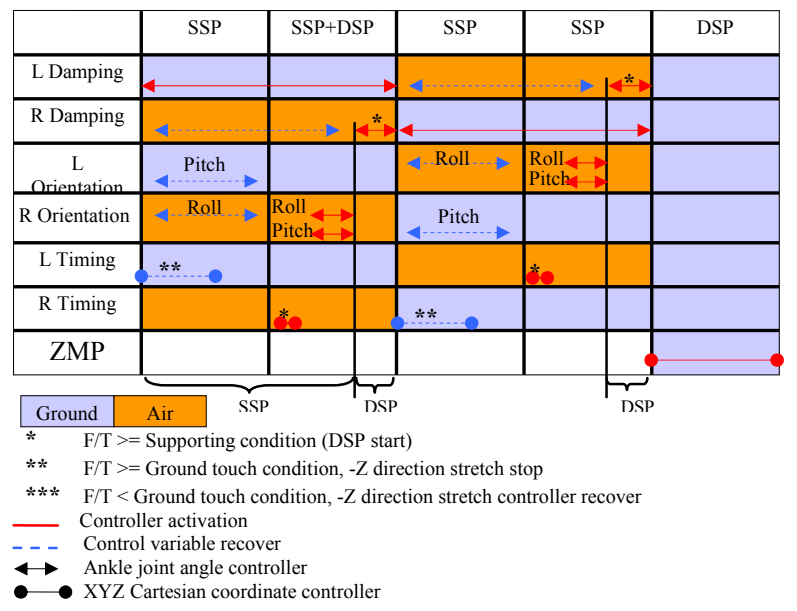

Fig. 12 Controller timing diagram (Right foot starting case) 


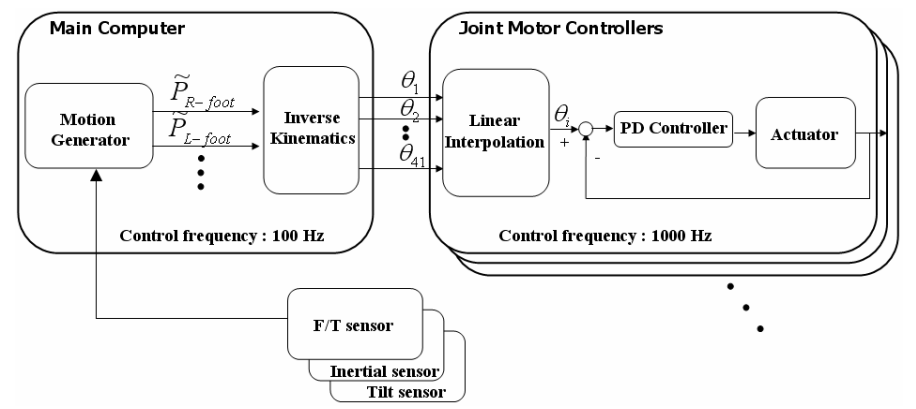

Fig 13 Motion control process in KHR-3

\section{HARDWARE DESCRIPTION}

We developed biped humanoid robot KHR-3 since 2004 shown in Fig. 14. Its height and weight is $125 \mathrm{~cm}$ and $55 \mathrm{Kg}$. It has 41-DOF (12-DOF in leg, 8-DOF in arm, 6-DOF in head, $14-\mathrm{DOF}$ in hand and $1-\mathrm{DOF}$ in trunk). It has $\mathrm{F} / \mathrm{T}$ sensors in the ankles and wrists, CCD-cameras in the head, tilt sensors to measure the inclination of ground in the feet, and inertia sensor in the trunk. It has distributed control hardware system based on CAN communication.

The robot has been upgraded from KHR-2. Its mechanical stiffness of links and reduction gear capacity of joints have been modified and improved. Increased stiffness makes it have low uncertainty of joint position and link vibration, which affect its stability. The joint controller, motor drive, battery, sensors and main controller (PC) are designed to be installed in the robot itself. All servo controller units for each actuator and all sensors are connected with CAN communication as shown in Fig. 15. The overall specification of KHR - 3 is given in Table I.

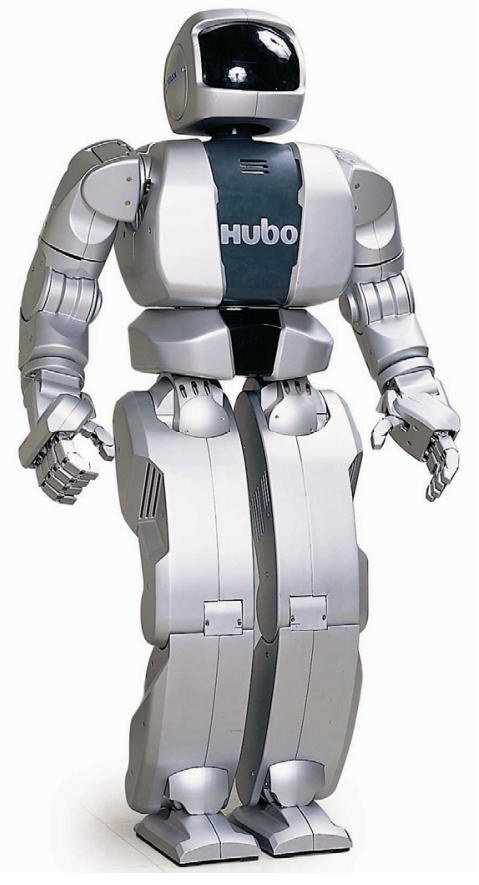

Fig. 14 Humanoid robot KHR-3(HUBO)

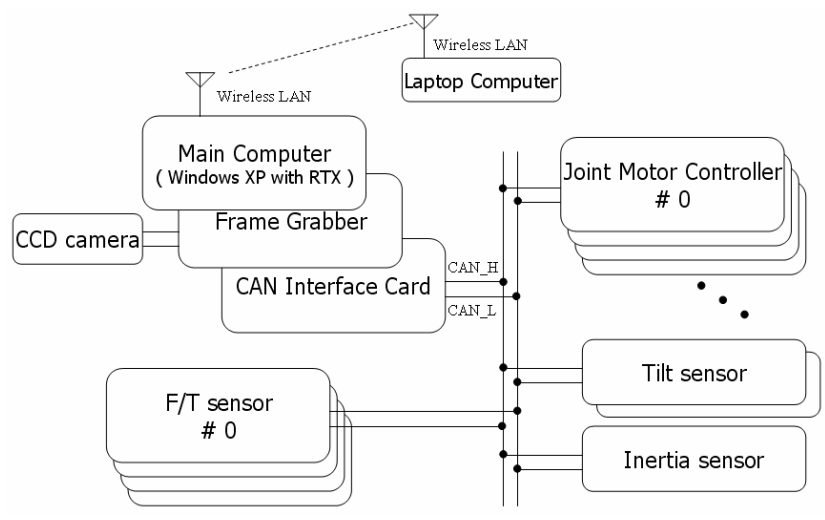

Fig. 15 System configuration of KHR-3(HUBO)

TABLE I

SPECIFICATION OF KHR-3

\begin{tabular}{|c|c|c|}
\hline \multicolumn{2}{|c|}{ Research term } & $2004.1 \sim$ \\
\hline \multicolumn{2}{|c|}{ Weight } & $55 \mathrm{Kg}$ \\
\hline \multicolumn{2}{|l|}{ Height } & $1.25 \mathrm{~m}$ \\
\hline \multicolumn{2}{|c|}{ Walking Speed } & $0 \sim 1.25 \mathrm{Km} / \mathrm{h}$ \\
\hline \multicolumn{2}{|c|}{ Walking Cycle, Stride } & $0.75 \sim 0.95 \mathrm{~s}, 0 \sim 64 \mathrm{~cm}$ \\
\hline \multicolumn{2}{|c|}{ Grasping Force } & $0.5 \mathrm{Kg} /$ finger \\
\hline \multicolumn{2}{|c|}{ Actuator } & $\begin{array}{l}\text { Servo motor + Harmonic Speed Reducer } \\
+ \text { Drive Unit }\end{array}$ \\
\hline \multicolumn{2}{|c|}{ Control Unit } & $\begin{array}{l}\text { Walking Control Unit, Servo Control Unit, } \\
\text { Sensor Communication Unit, } \\
\text { Communication Unit }\end{array}$ \\
\hline \multirow[t]{2}{*}{ Sensors } & Foot & 3-Axis Force Torque Sensor, Inclinometer \\
\hline & Torso & Rate Gyro \& Inclination Sensor \\
\hline \multirow{2}{*}{$\begin{array}{l}\text { Power } \\
\text { Section }\end{array}$} & Battery & $24 \mathrm{~V} / 3.3 \mathrm{Ah}-2 \mathrm{EA}, 12 \mathrm{~V} / 6.6 \mathrm{Ah}-$ \\
\hline & $\begin{array}{l}\text { External } \\
\text { Power }\end{array}$ & $\begin{array}{l}\text { 12V, 24V (Battery and External Power } \\
\text { Supply Changeable) }\end{array}$ \\
\hline \multicolumn{2}{|c|}{ Operation Section } & Laptop computer with wireless LAN \\
\hline \multicolumn{2}{|c|}{ Operating System (OS) } & Windows XP and RTX \\
\hline \multicolumn{2}{|c|}{ Degree of Freedom } & $41 \mathrm{DOF}$ \\
\hline
\end{tabular}

\section{EXPERIMENT}

We had an experiment of marking time with variable walking period on the floor. The purpose of this experiment is to realize the gait generation method stated in chapter I. The trajectory has the continuity and the smoothness for those periods as shown in Fig. 16. The periods that we used are 750, 850 and $950 \mathrm{~ms}$. Sy for each period is 47,60 , and $67 \mathrm{~mm}$ respectively. As the walking speed goes slow, we need to have large $S y$ to keep stability. We changed the period from 750 to $950 \mathrm{~ms}$ with $100 \mathrm{~ms}$ step at $4.875(\mathrm{~A}), 10.825(\mathrm{~B})$ and $16.525 \mathrm{~s}(\mathrm{C})$. We added $40 \%$ of $S y$ in starting step time $(0 \sim 750 \mathrm{~ms})$ because of the starting momentum effect. Fig. 17 shows the forward walking experiment result. We changed the step length of forward walking with $800 \mathrm{~ms}$ step time. The step lengths are $0 \mathrm{~mm}$ (start to $7.2 \mathrm{~s}), 70 \mathrm{~mm}(7.2 \mathrm{~s} \sim 14.4 \mathrm{~s}), 150 \mathrm{~mm}$ $(14.4 \mathrm{~s} \sim)$. It is shown that the continuity of the walking pattern with step length change is preserved. It is shown that there is an abnormal landing at time ' $A$ '. This situation happens when there is no landing signal is detected in despite of its fully stretched leg. The landing timing controller is activated at that time. All motion flows are halted and the controller waits the land OK signal. 


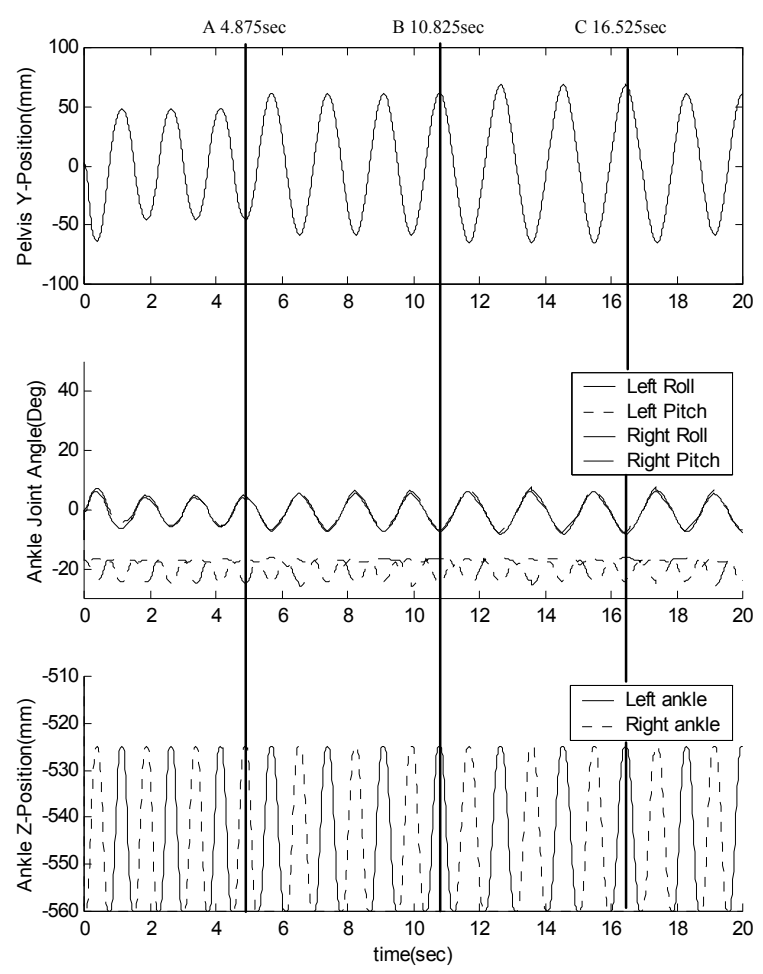

Fig. 16 Experimental result (Marking time)
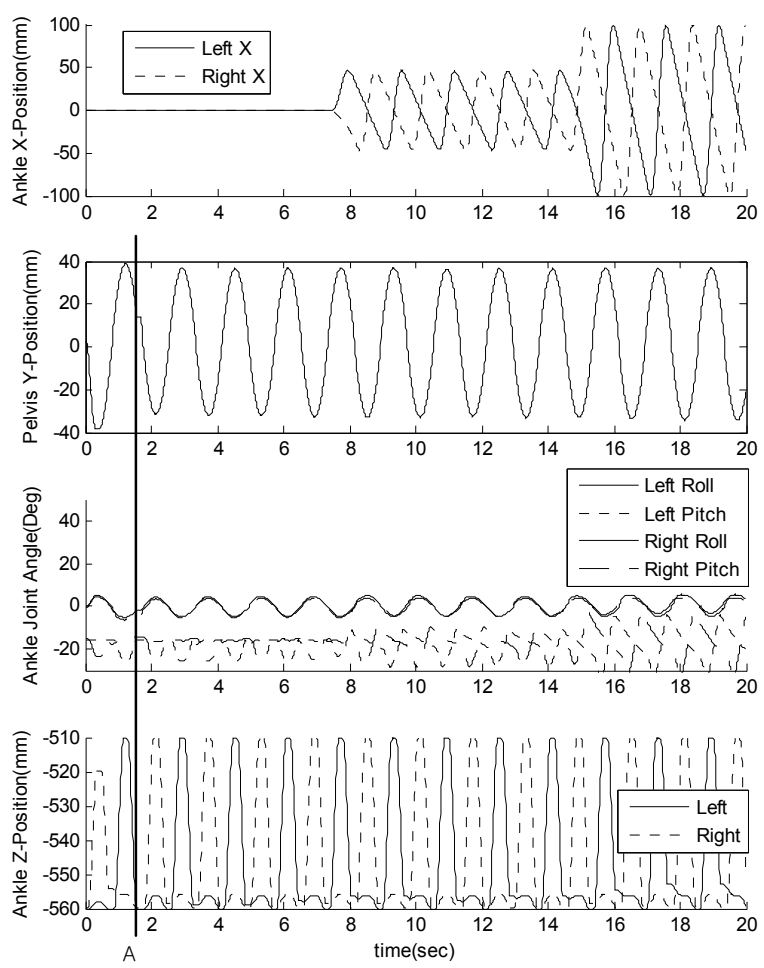

Fig. 17 Experimental result (Forward walk)

\section{CONCLUSION}

We proposed a novel online gait trajectory generation method which has the continuity in variable period and stride to realize the various bipedal walking gaits. It also has simple mathematic form and coordinates decoupled property. The method is proved by simulation and experiment, which we applied the control algorithm to the humanoid robot platform KHR-3.

\section{FUTURE WORK}

In this paper, we implemented the gait generation algorithm of marking time mode on the robot. We will realize various walking modes such as non-crossing gait ( $t_{0}$ does not exist case.) with variable frequency and stride. This is important for the free walking gait.

\section{ACKNOWLEDGEMENT}

This research was mainly supported by MOCIE (Ministry of Commerce, Industry and Energy) and partly supported by HWRS (Human Welfare Robotic System) and BK-21 (Brain Korea - 21) project.

\section{REFERENCES}

[1] K. Hirai, Current and Future Perspective of Honda Humanoid Robot, in Proc. IEEE/RSJ Int. Conference on Intelligent Robots and Systems, pp. 500-508, 1997

[2] K. Hirai, M. Hirose, Y. Haikawa, and T. Takenaka, The Development of Honda Humanoid Robot, in Proc. IEEE Int. Conf. on Robotics and Automations, pp.1321-1326, 1998

[3] Y. Sakagami, R. Watanabe, C. Aoyama, S. Matsunaga, N. Higaki, and K. Fujimura, The intelligent ASIMO: System overview and integration, in Proc. IEEE/RSJ Int. Conf. on Intelligent Robots and Systems, pp. 24782483, 2002

[4] J. Yamaguchi, A. Takanishi, and I. Kato, Development of a Biped Walking Robot Compensating for Three-Axis Moment by Trunk Motion, in Proc. IEEE/RSJ Int. Conf. on Intelligent Robots and Systems, pp.561-566, 1993

[5] H. Lim, Y. Kaneshima, and A.Takanishi, Online Walking Pattern Generation for Biped Humanoid Robot with Trunk, in Proc. IEEE Int. Conf. on Robotics \& Automation, pp. 3111-3116, 2002

[6] K. Nishiwaki, T. Sugihara, S. Kagami, F. Kanehiro, M. Inaba, and H. Inoue, Design and Development of Research Platform for PerceptionAction Integration in Humanoid Robot: H6, in Proc. IEEE/RJS Int. Conf. on Intelligent Robots and Systems, pp.1559-1564, 2000

[7] S. Kagami, K. Nishiwaki, J. J. Kuffner Jr., Y. Kuniyoshi, M. Inaba, and H. Inoue, Online 3D Vision, Motion Planning and Biped Locomotion Control Coupling System of Humanoid Robot: H7, in Proc. IEEE/RSJ Int. Conf. on Intelligent Robots and Systems, pp. 2557-2562, 2002

[8] K. Kaneko, F. Kanehiro, S. Kajita, K. Yokoyama, K. Akachi, T. Kawasaki, S. Ota, and T. Isozumi, Design of Prototype Humanoid Robotics Platform for HRP, in Proc. IEEE Int. Conf. on Inteligent Robots and Systems, pp.2431-2436, 1998

[9] S. Kajita, F. Kanehiro, K Kaneko, K Fujiwara, K Harada, K Yokoi, and H. Hirukawa, Biped Walking Pattern Generation by using Preview Control of Zero-Moment Point, in Proc. IEEE Int Conf. on Robotics \& Automation, pp. 1620-1626, 2003

[10] Jung-Hoon Kim and Jun-Ho Oh, Realization of dynamic walking for the humanoid robot platform KHR-1, Advanced Robotics, Vol. 18, no. 7, pp. 749-768, 2004

[11] Jung-Yup Kim, Ill-Woo Park, and Jun-Ho Oh, Design and Walking Control of the Humanoid Robot, KHR-2, International Conference on Control, Automation and Systems, pp. 1539-1543, 2004

[12] Jung-Yup Kim, Ill-Woo Park, Jungho Lee, Min-Su Kim, Baek-Kyu Cho and Jun-Ho Oh, "System Design And Dynamic Walking Of Humanoid Robot KHR-2", IEEE International Conference on Robotics \& Automation, 2005. 